\title{
Recent Advances in Improvement of Forecast Skill and Understanding Climate Processes Using AIRS Version-5 Products
}

\author{
Joel Susskind ${ }^{1}$, Gyula Molnar², Lena Iredell ${ }^{3}$, and Robert Rosenberg ${ }^{3}$ \\ ${ }^{1}$ NASA Goddard Space Flight Center, Greenbelt, MD, USA 20771 \\ ${ }^{2}$ Morgan State Univ./GESTAR, NASA Goddard Space Flight Center, Greenbelt, MD, USA 20771 \\ ${ }^{3}$ SAIC, NASA Goddard Space Flight Center, Greenbelt, MD, USA 20771
}

\begin{abstract}
The NASA Goddard Earth Sciences (GES) Data and Information Services Center (DISC) generates products derived from AIRS/AMSU-A observations, starting from September 2002 when the AIRS instrument became stable, using the AIRS Science Team Version-5 retrieval algorithm. This paper shows results of some of our research using Version-5 products from the points of view of improving forecast skill as well as aiding in the understanding of climate processes.

Keywords: AIRS/AMSU, high spectral resolution IR sounders, retrieval methodology, IR sounding in cloudy conditions, clear column radiances, quality control, data assimilation, climate processes, El Niño, OLR.
\end{abstract}

\section{INTRODUCTION}

The Atmospheric Infrared Sounder (AIRS) was launched on EOS Aqua in May 2002, together with the Advanced Microwave Sounding Unit (AMSU-A) and the Humidity Sounder for Brazil (HSB) (which subsequently failed early in the mission), to form a next generation polar orbiting infrared and microwave atmospheric sounding system ${ }^{1}$. AIRS/AMSU had two primary objectives. The first objective was to provide real-time data products to be used in a data assimilation mode by operational Numerical Weather Prediction Centers so as to improve the skill of their subsequent forecasts. The second objective was to provide accurate unbiased sounding products with good spatial coverage that are used to generate stable multi-year climate data sets used to study the earth's interannual variability, climate processes, and possibly long-term trends.

AIRS is a grating spectrometer with a number of linear arrays of detectors with each detector sensitive to outgoing radiation in a characteristic frequency $v_{i}$ with a spectral band pass $\Delta v_{i}$ of roughly $v_{i} / 1200$. AIRS contains 2378 spectral channels covering portions of the spectral region $650 \mathrm{~cm}^{-1}(15.38 \mu \mathrm{m})$ to $2665 \mathrm{~cm}^{-1}$ (3.752 $\left.\mu \mathrm{m}\right)$. The spectral sampling interval (except for the existence of a few gaps) is $v_{i} / 2400$, giving two AIRS channels per spectral half width. AIRS is accompanied by the temperature sounding $60 \mathrm{GHz}$ microwave instrument AMSU-A. There is a 3x3 array of AIRS footprints within a given AMSU-A footprint, with spatial resolutions of $13 \mathrm{~km}$ and $45 \mathrm{~km}$ at nadir viewing respectively. Each AIRS footprint is referred to as a Field of View (FOV), and the AMSU-A footprint is referred to as a Field of Regard (FOR).

The NASA Goddard Earth Sciences (GES) Data and Information Services Center (DISC) generates products derived from AIRS/AMSU-A observations, starting from September 2002 when the AIRS instrument became stable, using the AIRS Science Team Version-5 retrieval algorithm. ${ }^{2}$ The methodology used to analyze AIRS/AMSU observations is essentially unchanged from that described in Susskind et al. (2003) ${ }^{3}$. Fundamental to this approach is the generation of clear column radiances for each AIRS channel $i, \hat{R}_{i}$, which are derived products representing the radiance channel $i$ would have seen if the entire 3x3 AIRS FOR were cloud free. $\hat{R}_{i}$ is determined for each channel as a linear combination of the observed radiances of that channel in each of the 9 FOV's, using coefficients that are channel independent. The retrieved geophysical state $X$ is subsequently determined which, when substituted in a Radiative Transfer Algorithm (RTA), generates an ensemble of computed radiances $R_{i}(X)$ which are consistent with $\hat{R}_{i}$ for those channels i used in the determination of $X$. Cloud-clearing theory ${ }^{4,5}$ says that to achieve the best results in more stressing cloud conditions, longwave channels sensitive to cloud contamination should be used only in the determination of the coefficients used in the generation of clear column radiances for all channels and not for sounding purposes. In Version- $5^{2}$, tropospheric 
sounding $15 \mu \mathrm{m} \mathrm{CO}_{2}$ observations were used only in the derivation of the cloud clearing coefficients, and temperature profiles were derived using $\hat{R}_{i}$ in the $4.3 \mu \mathrm{m} \mathrm{CO}$ band as well as in some stratospheric $15 \mu \mathrm{m} \mathrm{CO}$ channels that do not see clouds. This new approach allowed for the retrieval of accurate quality controlled values of $\hat{R}_{i}$ and $T(p)$ under more stressing cloud conditions than was achievable in Version-4. Version-5 also contained a new methodology, compared to that of Version-4, to provide accurate case-by-case error estimates for retrieved geophysical parameters and for channelby-channel clear column radiances. Thresholds of these error estimates were used in a new approach for quality control.

This paper shows recent examples of research that has been conducted in terms of assimilation of quality controlled AIRS Science Team Version-5 Level-2 case-by-case retrievals to improve numerical weather prediction forecast skill, as well as research using AIRS Version-5 multiyear Level-3 gridded products to study climate processes.

\section{FORECAST IMPACT EXPERIMENTS USING QUALITY CONTROLLED VERSION-5 TEMPERATURE PROFILES}

AIRS Version-5 retrievals contain case-by-case level-by-level error estimates for all accepted profiles ${ }^{2}$. These error estimates are used to determine a case-by-case characteristic pressure $p_{\text {best }}$, down to which the profile is considered acceptable. All accepted profiles are assigned to have high quality down to at least $70 \mathrm{mb}$. A characteristic pressure $p_{\text {best }}$ is defined as the highest pressure (somewhere between $70 \mathrm{mb}$ and the surface pressure) at which the error estimate is not greater than a pressure dependent error estimate threshold. The Version-5 pressure dependent thresholds, called Standard Quality Control thresholds, were optimized bearing in mind what was considered to be the best trade-off between accuracy and spatial coverage for use in both data-assimilation and climate applications. Data assimilation, in general, requires high accuracy retrievals, while climate studies require good spatial coverage with less accurate, but unbiased, retrievals. Spatial coverage and accuracy of Version-5 retrievals using Standard Quality Control thresholds, as well as those using Tighter Quality Control thresholds, are shown in Susskind et al. (2011). ${ }^{2}$

We conducted a number of data assimilation experiments using AIRS Version-5 quality controlled temperature profiles as a step toward finding an optimum balance of spatial coverage and sounding accuracy with regard to improving forecast skill. The data assimilation and forecast system we used was the Goddard Earth Observing System Model , Version-5 (GEOS-5) Data Assimilation System (DAS), which represents a combination of the NASA GEOS-5 forecast model with the National Centers for Environmental Prediction (NCEP) operational Grid Point Statistical Interpolation (GSI) global analysis scheme. All analyses and forecasts were run at a $0.5^{\circ} \mathrm{x} 0.625^{\circ}$ spatial resolution. Data assimilation experiments were conducted in four different seasons, each in a different year. The four periods studied were January 1 to January 31, 2003; October 10 to November 19, 2005; August 10 to September 16, 2006; and April 15 to May 18, 2008. Seven day forecasts were run every day in each experiment, beginning several days after the start of each experiment to allow for spin up. The forecasts were verified every 12 hours against the NCEP analysis, which was taken as "truth".

Four different sets of data assimilation experiments were run during each time period: Control; AIRS Standard; AIRS Tight; and Radiance. In the "Control" analysis, all the data used operationally by NCEP was assimilated, but no AIRS data was assimilated. Radiances from the Aqua AMSU-A instrument were also assimilated operationally by NCEP and are included in the "Control". It should be noted that the Aqua orbit (1:30 p.m. ascending) is almost identical to that of National Oceanic and Atmospheric Administration (NOAA)-16 carrying High-resolution Infrared Sounder3, AMSU-A, and AMSU-B. Therefore, AIRS/AMSU temperature soundings, if used, provide additional information to that contained in the AMSU-A/AMSU-B radiances on NOAA-16 in the same orbit, as well as those of the Aqua AMSU-A radiances. No AIRS data of any kind were assimilated operationally at that time. In AIRS Standard and AIRS Tight Assimilations, all information used in the Control was assimilated as well as quality controlled AIRS Version-5 temperature profiles. The AIRS Version-5 temperature profiles were presented to the GSI analysis as rawinsonde profiles, assimilated down to appropriate pressure level $p_{\text {best }}$. The case-by-case level-by-level error estimates of the temperature profiles were used as the uncertainty of each temperature measurement.

NCEP and European Center for Medium-Range Weather Forecasts (ECMWF) now assimilate AIRS observations operationally. The current operational practice is to directly assimilate observed AIRS radiances rather than AIRS temperature soundings. The operational methodologies used by both NCEP and ECMWF do not have the capability to 
derive and assimilate cloud cleared AIRS radiances. Instead, the analysis procedures used at both Centers select and assimilate only those AIRS observations which are "thought to be unaffected by clouds.” These uncontaminated radiance observations are influenced primarily by temperatures in the stratosphere and also above clouds in areas where clouds are present. Our results from AIRS indicate that roughly 95\% of AIRS pixels are cloud contaminated. Therefore, information from most tropospheric sounding AIRS observations is not included in the operational AIRS radiance assimilation process. In the Radiance Assimilation experiment, we assimilated AIRS radiances according to the NCEP operational procedure. In these AIRS Radiance Assimilation experiments, all other data assimilated in the Control was also included, but no AIRS temperature profile data was assimilated.

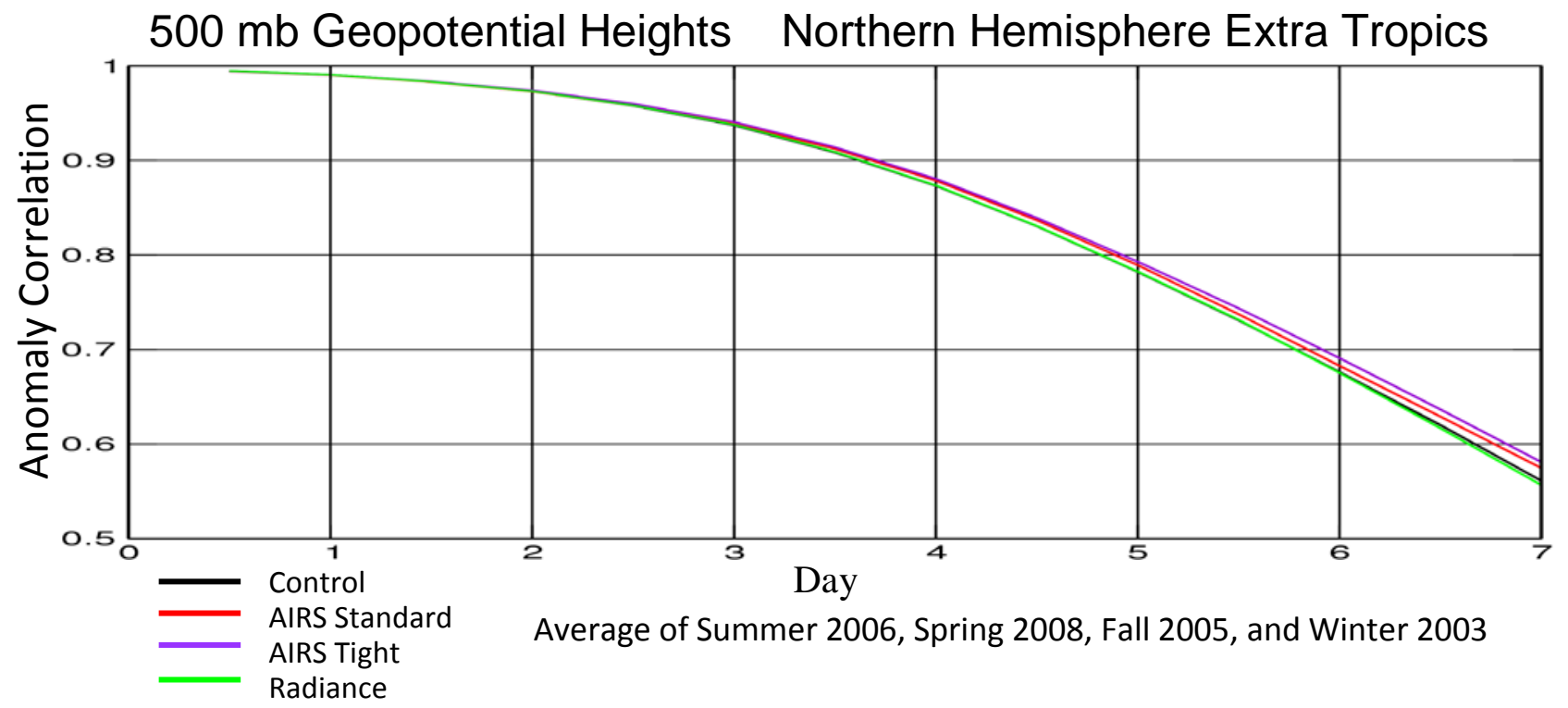

Fig. 1

Figure 1 shows the average over all the experiments, of the 12-hour to seven day forecast $500 \mathrm{mb}$ Geopotential Height anomaly correlation coefficients verified against the NCEP analysis for the Northern Hemisphere extra-tropics. An anomaly correlation of 1.0 represents a perfect forecast and an anomaly correlation of 0.6 represents the limit of what is considered to be a useful forecast. An improvement in forecast skill of one experiment compared to another is indicated by the increase in hours (shift to the right) for that forecast to have the equivalent skill compared to another. In the Northern Hemisphere Extra-Tropics, assimilating quality controlled AIRS soundings resulted in an improvement in average seven day forecast skill of roughly five hours compared to the Control for the Tight AIRS assimilation, and four hours for the AIRS Standard Assimilation.

Assimilation of AIRS radiances unaffected by clouds resulted in a substantially reduced forecast impact in the Northern Hemisphere Extra-Tropics, compared to the Control, than that obtained by assimilating AIRS temperatures. At least a part of this loss in forecast impact of Radiance Assimilation in the Northern Hemisphere Extra-Tropics results from the significant loss of spatial coverage of the AIRS tropospheric sounding channels used in the data assimilation process due to cloud contamination. In the Southern Hemisphere Extra-Tropics (not shown), seven day forecasts from the Radiance Assimilation again produced essentially no improvement compared to the Control, while forecasts from the AIRS Tight Assimilation resulted in about a two hour improvement in average forecast skill compared to the Control, and forecasts from the Standard Assimilation resulted in a two hour degradation of forecast skill compared to the Control, and a four hour degradation compared to those from the Tight Assimilation. This demonstrates the importance of using appropriate quality control when assimilating the AIRS temperature profiles.

Even more significant than the improvement in 7-day forecast skill is the finding that assimilation of AIRS temperature soundings results in a significant improvement in the depiction of severe tropical weather systems and the subsequent ability to predict storm tracks for these events. We studied eight intense tropical cyclone events in detail which took place during the time of the four data assimilation experiments conducted. We found that in each case, the AIRS Tight Analysis improved the depiction of the tropical cyclones in the GEOS-5 DAS with regard to their intensity, confinement 
and position. The cause of the improvements was the ability to detect tight, strong upper-tropospheric positive thermal anomalies over areas of organized convection. In all cases, we obtained a much better prediction of the location and time of landfall of these tropical cyclones using forecasts from the AIRS Tight Assimilation compared to those obtained using either the Control or Radiance Assimilation analyses. An important example is given in Reale et al. ${ }^{6}$, which showed the ability to accurately predict landfall five days ahead of time for Tropical Cyclone Nargis, which devastated parts of Myanmar with considerable loss of life in May 2008. This storm track was not predicted accurately from either the Control or Radiance Assimilation analyses. Zhou et al. ${ }^{7}$ also showed that assimilation of quality controlled AIRS temperatures led to significantly improved precipitation analyses and forecasts for three tropical cyclones compared to what was obtained using the Control or Radiance Assimilations. We recently conducted another analogous Data Assimilation experiment covering the period July 15, 2010 through August 30, 2010. We chose the period to cover the set of catastrophic rain episodes that occurred in late July 2010 along the Indus Valley, in central and northern Pakistan. Reale et al. ${ }^{8}$ showed that in addition to improving the global forecast skill, the assimilation of quality-controlled AIRS temperature retrievals obtained under partly cloudy conditions produce better precipitation analyses than assimilation of radiances unaffected by clouds.

Our experiments using GEOS-5 indicate the potential to improve operational forecasting skill exists by the assimilation of quality controlled AIRS temperature profiles rather than AIRS radiances as is currently done operationally. In order to test if this is indeed the case, we ported the NCEP Operational Data Assimilation System (GDAS) to GSFC. We started conducting preliminary experiments at GSFC, assimilating AIRS Version-5 quality controlled temperature profiles into the NCEP operational GDAS system and comparing the results to those using the operational procedure of assimilating AIRS radiances, and also to a Control run which assimilated no AIRS data. These preliminary experiments were conducted starting from July 15, 2010, just as those studied by Reale et al. ${ }^{8}$.

As of this writing, the AIRS Science Team Version-6 retrieval algorithm has been finalized and should become operational at the GES DISC in late 2012. The AIRS Version-6 retrieval algorithm has further advances over Version-5. These modifications have resulted in significant improvement in the ability to obtain both accurate temperature profiles and surface skin temperatures under more stressing partial cloud cover conditions. ${ }^{9}$ These improvements will enhance the utility of AIRS retrievals both from the perspectives of improving numerical weather prediction as well as understanding climate processes. We plan in the near future to also conduct analogous experiments using GDAS for the period January 1 to 31, 2003, assimilating both Version-5 retrievals as well as Version-6 retrievals, which have already been run in a test mode for that month.

\section{THE EFFECT OF EL NIÑO/LA NIÑA OSCILLATIONS ON TROPICAL CIRCULATION AND OLR}

Outgoing Longwave Radiation (OLR) represents the total thermal radiation emitted by the earth-atmospheric system and transmitted to space. OLR is a critical part of the Earth's radiation balance which maintains the earth's thermal stability. Low values of OLR in the tropics have also been used as a proxy for convective activity and precipitation. OLR has been determined from satellite using a sequence of instruments measuring broad band spectral fluxes. The most recent of these is Clouds and Earth's Radiant Energy System (CERES) ${ }^{10}$ instrument, which is flying on EOS Aqua and EOS Terra as well as on other satellites. OLR is also a product generated by the AIRS Science Team which is computed as a function of other geophysical parameters derived from AIRS/AMSU observations. This study compares anomaly time series of OLR derived independently from CERES and AIRS observations and uses other AIRS derived products to explain the recent behavior of OLR as observed by both CERES and AIRS.

For the purposes of this study, we use the operational monthly mean OLR data products produced by the AIRS and CERES Science Teams. We obtained the AIRS OLR products from the GES DISC and the CERES products from the CERES Science Team website. We use the CERES Terra OLR products generated by the CERES Science Team because these extended further in time than those of CERES Aqua OLR. AIRS was launched on the EOS Aqua satellite in a 1:30 AM/PM local crossing time orbit in May 2002. The operational processing of AIRS data began after AIRS became stable in September 2002. We use the AIRS Version-5 monthly mean Level-3 $1^{\circ}$ x $1^{\circ}$ latitude-longitude grid products which contain separate products generated for each of the 1:30 AM and PM local time orbits. We averaged the AM and PM products together to generate and use a single monthly mean product on the $1 \times 1^{\circ}$ grid for each month. In addition to 
AIRS OLR, we also use the AIRS Level-3 surface skin temperatures, water vapor profiles, and cloud products to demonstrate the behavior of factors contributing significantly to the anomaly time series of OLR.

\subsection{Computation of AIRS OLR as a function of surface and atmospheric conditions}

OLR at a given location is affected primarily by the earth's skin surface temperature, $T_{s}$; skin surface spectral emissivity, $\epsilon_{v}$; atmospheric vertical temperature profile $T(p)$ and water vapor profile, $q(p)$; and the heights, amounts, and spectral emissivities of multiple layers of cloud cover. OLR also depends on the vertical distributions of trace gases such as $\mathrm{O}_{3}(p), \mathrm{CH}_{4}(p), \mathrm{CO}_{2}(p)$, and $\mathrm{CO}(p)$. AIRS products are generated for each AIRS FOR. OLR is computed for a specific AIRS FOR, given all the needed geophysical parameters, using an OLR Radiative Transfer Algorithm (RTA). ${ }^{11}$ The AIRS results shown in this paper were derived using the AIRS Science Team Version-5 retrieval algorithm which generates the values of $T_{s}, \epsilon_{v}, T(p), q(p), O_{3}(p)$, and cloud parameters, from which OLR and OLR CLR $_{\text {(clear-sky OLR) are }}$ computed. The AIRS Version-5 OLR product, referred to as $F$ below, is computed as a sum of fluxes in 14 contiguous spectral bands according to

$$
F=\sum_{j=1}^{14} F_{j}=\sum_{j=1}^{14}\left(1-\alpha \varepsilon_{1 j}-\alpha \varepsilon_{2 j}\right) F_{j, C L R}+\alpha \varepsilon_{1 j} F_{j, C L D 1}+\alpha \varepsilon_{2 j} F_{j, C L D 2}
$$

where $F_{j, C L R}$ is the computed clear sky flux going to space integrated over all angles emanating from spectral band $j$; $F_{j, C L D k}$ is the analogous computed flux emanating from an opaque cloud at cloud top pressure $p_{k}$; and $\alpha \varepsilon_{k j}$ is the radiatively effective cloud fraction given by the product of the geometric fractional cloud cover $\alpha_{k}$ as seen from above for the cloud at pressure $p_{k}$ and the emissivity of that cloud in spectral band $j . F_{j, C L R}$ is computed according to

$$
F_{j, C L R}=\pi\left[\varepsilon_{j} B\left(v_{j}, T_{s}\right) \tau_{j}\left(p_{s}\right)+\int_{\ell n p_{s}}^{\ell n \bar{p}} B\left(v_{j}, T(p)\right) \frac{d \tau_{j}}{d \ell n p} d \ell n p\right]
$$

where $B(v, T)$ is the Planck Blackbody function evaluated at frequency $v$ and temperature $T, v_{j}$ is the central frequency of spectral band $j, \varepsilon_{j}$ is the surface emissivity in band $j$, and the term $\tau_{j}(p)$ represents the effective band averaged atmospheric transmittance in band $j$ from pressure $p$ to the top of the atmosphere $\bar{p}$. To first order, the integral in Equation 2 can be approximated by $B\left(v_{j}, T\left(p_{j}\right)\right) \times\left(1-\tau_{j}\left(p_{s}\right)\right)$ where $p_{j}$ is a band effective pressure for which $\tau_{j}\left(p_{j}\right)=e^{-1} \cdot F_{j, C L D}$ is computed in an analogous way to $F_{j, C L R}$, but $T_{s}$ is replaced by $T\left(p_{k}\right)$ and $p_{s}$ is replaced by $p_{k}$ in Equation 2. According to Equation 2, everything else being held constant, $F$ increases with increasing temperature which increases both the surface and atmospheric contributions in Equation 2. In addition, Equation 2 shows that $F$ decreases with increasing atmospheric water vapor which decreases $\tau_{j}(p)$ in bands sensitive to water vapor absorption, especially for very moist (tropical cases). $F$ also decreases with increasing $\alpha \varepsilon$, especially for high (cold) clouds. More details about the comparison of AIRS and CERES OLR products are given in Susskind et al. (2012) ${ }^{12}$.

\subsection{Comparison of AIRS and CERES global mean and tropical mean anomaly time series}

We generated AIRS and CERES monthly mean OLR and OLR $_{\text {CLR }}$ climatologies on a $1^{\circ}$ x $1^{\circ}$ spatial resolution for each month of the year by taking the average of the grid box value for that month over an eight-year time period, i.e., eight consecutive Januaries, eight consecutive Februaries, etc. The same ensembles of eight Januaries, Februaries, etc., are used in the generation of climatologies for all products shown in this paper. OLR and OLR $_{C L R}$ anomalies for a given month in a given year, on a $\mathrm{P} \times 1^{\circ}$ spatial grid, are defined as the differences between their monthly mean values in that year and their monthly climatologies for that grid box. The area mean anomaly for a given month and year is defined as the cosine latitude weighted average of the grid box anomalies contained in the area under consideration.

Figure 2a shows the global mean anomaly time series of AIRS Version-5 OLR and CERES OLR for the period September 2002 through June 2011, as well as the difference between the two sets of monthly mean anomalies. Figure 2b shows analogous results for tropical mean OLR anomalies. We define the term El Niño Index as the difference of the NOAA monthly mean oceanic Sea Surface Temperature (SST), averaged over the NOAA El Niño spatial area $5^{\circ} \mathrm{N}$ to $5^{\circ} \mathrm{S}$ latitude and $150^{\circ} \mathrm{W}$ westward to $160^{\circ} \mathrm{E}$ longitude, from an eight year NOAA El Niño SST monthly mean climatology we generated based on eight years of data in an analogous manner to that used in the generation of the OLR climatologies. 


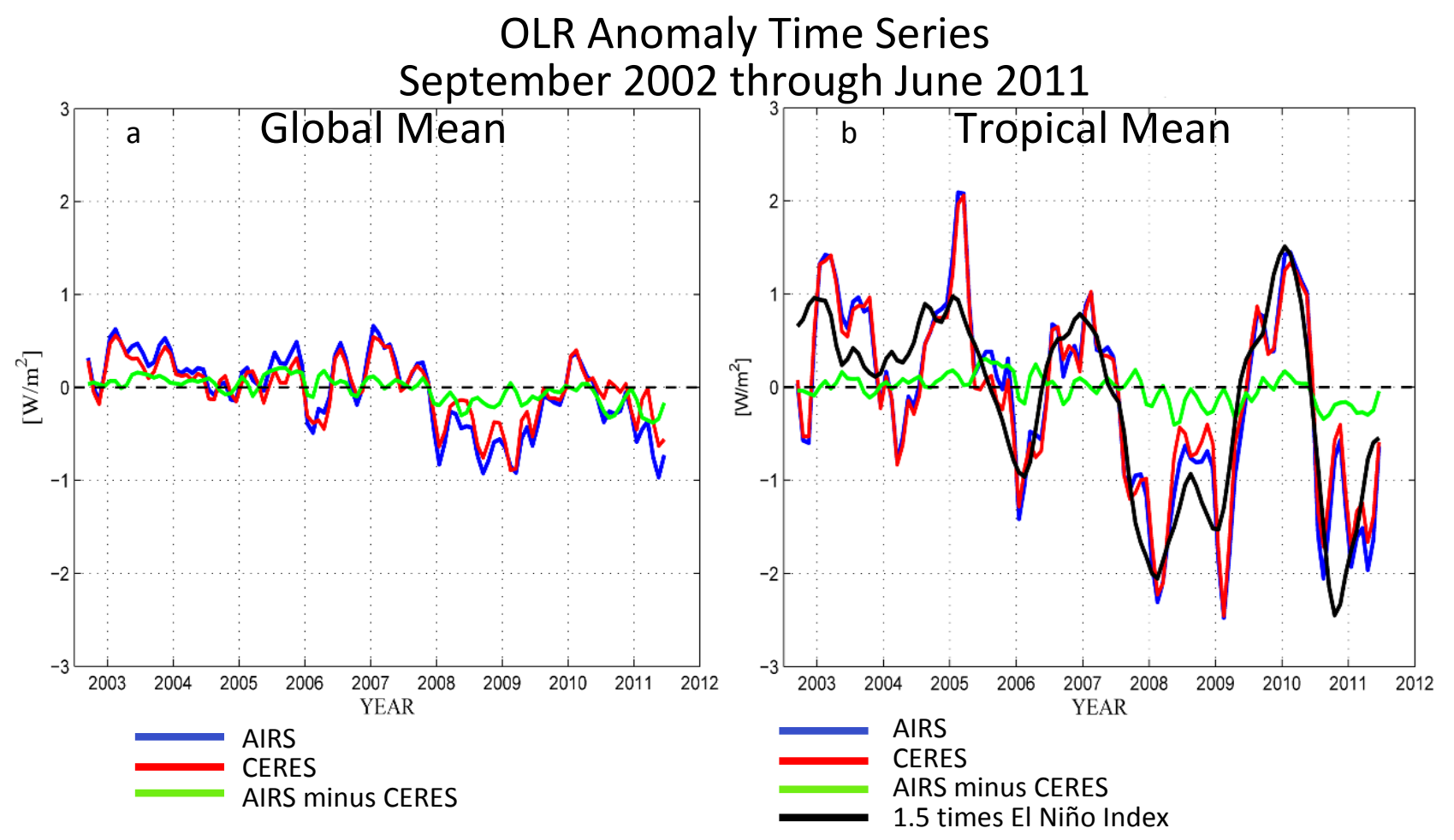

Fig. 2

Figures $2 \mathrm{~b}$ includes the values of the El Niño Index multiplied by 1.5. Tropical mean OLR anomalies both tend to track those of the El Niño Index in phase fairly closely. Positive values of the El Niño Index (2003, 2005, 2007, early 2010) correspond to El Niño (positive SST anomalies in the El Niño area) periods, and negative values (2008, mid-2010 to the present) correspond to La Niña (negative SST anomalies) periods. Figure 2a shows that an onset of negative global mean anomalies for OLR began in late 2007. The negative tropical mean anomalies of OLR shown in Figure $2 \mathrm{~b}$ are generally considerably larger than the corresponding global mean anomalies, especially after mid-2007. The decreases in global mean OLR in late 2007 are strongly influenced by the significant reduction in tropical mean OLR which started a few months earlier. Tropical mean OLR anomalies became positive starting in late 2009, roughly coincident with the onset of another El Niño event. Very substantial negative global and especially tropical mean OLR anomalies occurred in the period starting mid-2010, when the latest La Niña event began.

We define the Average Rate of Change (ARC) of an anomaly time series as the slope of the linear least squares fit of the anomaly time series. We use the term Average Rate of Change to describe the slope of an anomaly time series rather than the term trend, which is generally used to characterize long-term multi-decadal data sets rather than the eight year 10 month period studied in this paper. Figure $2 \mathrm{~b}$ shows that the El Niño Index is highly non-linear over this time period, with fluctuating values that are primarily positive at the start of the time period and substantially negative at the end of the time period. The ARC of the El Niño Index, computed over the time period September 2002 through June 2011, is $-0.123 \pm 0.046 \mathrm{~K} / \mathrm{yr}$. The uncertainties shown here and subsequently represent twice the standard error, $\sigma$, of the regression slope of the linear least squares fit. The precise value of the ARC of the El Niño Index, which depends on the length of the time period used in the calculation, is less important than its sign, which shows that the El Niño region has cooled on the average over the time period under study.

Spatial distributions of ARCs of OLR and other geophysical parameters, to be shown later, are very coherent and are particularly informative with regard to the understanding of why global mean and tropical mean OLR decreased over the period September 2002 through June 2011. In this context, it is also very informative, if not more so, to examine the spatial distribution of temporal correlations of $1^{\circ} \times 1^{\circ}$ grid point anomaly tim e series with that of the El Niño Index. We refer to these temporal correlations of anomaly time series around the earth with the single anomaly time series of SST 
averaged over the NOAA El Niño region as El Niño Correlations (ENCs). ENCs represent both the phase and magnitude of the relationship between the time series of grid point anomalies and that of the El Niño Index. Unlike ARCs, ENCs should not depend significantly on the extent of the time series used to compute them if these correlations hold up over long time periods. In those spatial areas in which the ARCs of OLR are strongly influenced by El Niño/La Niño activity, there should be a very close agreement between the spatial patterns of ARCs of OLR with those of the ENCs of OLR and these patterns will be of opposite sign as a result of the negative ARC of the El Niño Index.

Table 1 shows global mean and tropical mean values of the ARCs of AIRS OLR and CERES Terra OLR anomalies over the time period September 2002 through June 2011, the standard deviations between the two sets of global mean and tropical mean anomaly time series, and the temporal correlations between each global mean and each tropical mean anomaly time series. All statistics use values of the three point smoothed anomaly time series shown in Figure 2. The agreement of the ARCs of both global mean and tropical mean anomaly time series found in the AIRS and CERES OLR records is on the order of $\pm 0.03 \mathrm{~W} / \mathrm{m}^{2} / \mathrm{yr}$, which is within the uncertainty of the respective sets of ARCs. The standard deviation between the AIRS and CERES OLR anomaly time series are on the order of $0.15 \mathrm{~W} / \mathrm{m}^{2}$ and the temporal correlations of the AIRS and CERES global mean and tropical mean OLR anomaly time series are 0.955 and 0.991 respectively. Both AIRS and CERES OLR anomaly time series show that global mean OLR has decreased on the average on the order of $-0.075 \mathrm{~W} / \mathrm{m}^{2} / \mathrm{yr}$ over the time period September 2002 through June 2011, and that tropical mean OLR has decreased at a rate of roughly $-0.168 \mathrm{~W} / \mathrm{m}^{2} / \mathrm{yr}$ from the beginning of the time period to the end. Demonstration of the ability to obtain close agreement between global and tropical mean ARCs of AIRS and CERES OLR anomaly time series, obtained in very different manners, is more significant than the values of the ARCs themselves, which are influenced by the extent of the time period used in the AIRS/CERES OLR data record comparison.

Table 1. OLR Anomaly Time Series Comparison

September 2002 through June 2011

\begin{tabular}{|l|c|c|}
\hline \multicolumn{1}{|c|}{ Data Set } & Global & Tropical \\
\hline AIRS ARC $\left(\mathrm{W} / \mathrm{m}^{2} / \mathrm{yr}\right)$ & $-0.094 \pm 0.026$ & $-0.183 \pm 0.070$ \\
\hline CERES Terra ARC $\left(\mathrm{W} / \mathrm{m}^{2} / \mathrm{yr}\right)$ & $-0.059 \pm 0.022$ & $-0.154 \pm 0.066$ \\
\hline AIRS Minus CERES STD $\left(\mathrm{W} / \mathrm{m}^{2}\right)$ & 0.136 & 0.155 \\
\hline AIRS/CERES Correlation & 0.955 & 0.991 \\
\hline
\end{tabular}

\subsection{The spatial distribution of ARCs and ENCs of OLR}

We show the global spatial distributions of OLR ARCs over the time period September 2002 through June 2011 in Figures $3 \mathrm{a}$ and $3 \mathrm{~b}$ for AIRS and CERES, respectively on a $1^{\circ}$ latitude by $1^{\circ}$ longitude grid. Figures $3 \mathrm{a}, \mathrm{b}$, and some subsequent figures, contain boxes surrounding three areas. The first box, shown in gray, surrounds the NOAA El Niño region, $5^{\circ} \mathrm{N}$ to $5^{\circ} \mathrm{S}$ and $150^{\circ} \mathrm{W}$ westward to $160^{\circ} \mathrm{E}$. A second box, shown in black, lies to the west of the El Niño region and encompasses the area between $20^{\circ} \mathrm{N}$ and $20^{\circ} \mathrm{S}$ from $90^{\circ} \mathrm{E}$ eastward to $135^{\circ} \mathrm{E}$. Much has been written about the meteorology of this region, which includes the Maritime Continent and the Western Pacific Warm Pool, as well as Darwin, Australia, particularly with regard to its response to El Niño activity. In particular, the Southern Oscillation Index (SOI), which represents monthly mean value of Tahiti sea level pressure minus that of Darwin, is well known to be highly negatively correlated with El Nino activity, and the two phenomena are often linked together with the single acronym El Niño/Southern Oscillation (ENSO). We refer to the area encompassed by this box as the Warm Pool Maritime Continent (WPMC) region. A third box, also shown in black, covers portions of the eastern tropical Pacific Ocean, northern South America, and the equatorial Atlantic Ocean and is the composite of three adjacent rectangles: $5^{\circ} \mathrm{N}$ to $20^{\circ} \mathrm{S}, 140^{\circ} \mathrm{W}$ to $95^{\circ} \mathrm{W} ; 8^{\circ} \mathrm{N}$ to $20^{\circ} \mathrm{S}, 95^{\circ} \mathrm{W}$ to $70^{\circ} \mathrm{W}$; and $8^{\circ} \mathrm{N}$ to $8^{\circ} \mathrm{S}, 70^{\circ} \mathrm{W}$ to $10^{\circ} \mathrm{E}$. We refer to the area encompassed by these three contiguous rectangles as the Equatorial Eastern Pacific and Atlantic (EEPA) region. Less attention has been paid to the meteorology of this region and its response to El Niño, especially that of the Atlantic Ocean. 
More significant than the precise values of the ARCs shown in Figure 3 is the very coherent spatial structure of the ARCs of OLR. Figures 3a and 3b demonstrate two very important points. The first is the virtually indistinguishable spatial distributions of the ARCs of AIRS OLR and of CERES OLR. The spatial correlation between the ARCs of the two OLR data sets is 0.98 , and the spatial standard deviation of the two sets of ARCs is $0.15 \mathrm{~W} / \mathrm{m}^{2} / \mathrm{yr}$. The global mean AIRS OLR ARC for this period is $0.035 \mathrm{~W} / \mathrm{m}^{2} / \mathrm{yr}$ lower (more negative) than that of CERES Terra. The most important scientific point of Figures $3 \mathrm{a}$ and $3 \mathrm{~b}$ is that while the global mean and tropical mean ARCs of OLR are negative, considerable spatially coherent longitudinal structure occurs in the ARCs of OLR at a given latitude, with differing signs and amplitudes. This structure is largest in the tropics, but is found at other latitudes as well. Figure 3 shows that positive OLR ARCs as large as $4.2 \mathrm{~W} / \mathrm{m}^{2} / \mathrm{yr}$ exist in the vicinity of the equatorial dateline, including and surrounding the El Niño region. These are more than compensated for, in the tropical mean sense, by negative OLR ARCs at other longitudes, as large as $-3.2 \mathrm{~W} / \mathrm{m}^{2} / \mathrm{yr}$ near the equator over Indonesia in the vicinity of $120^{\circ} \mathrm{E}$ longitude which lies within the WPMC

\section{OLR Average Rates of Change (ARCs) September 2002 through June 2011}

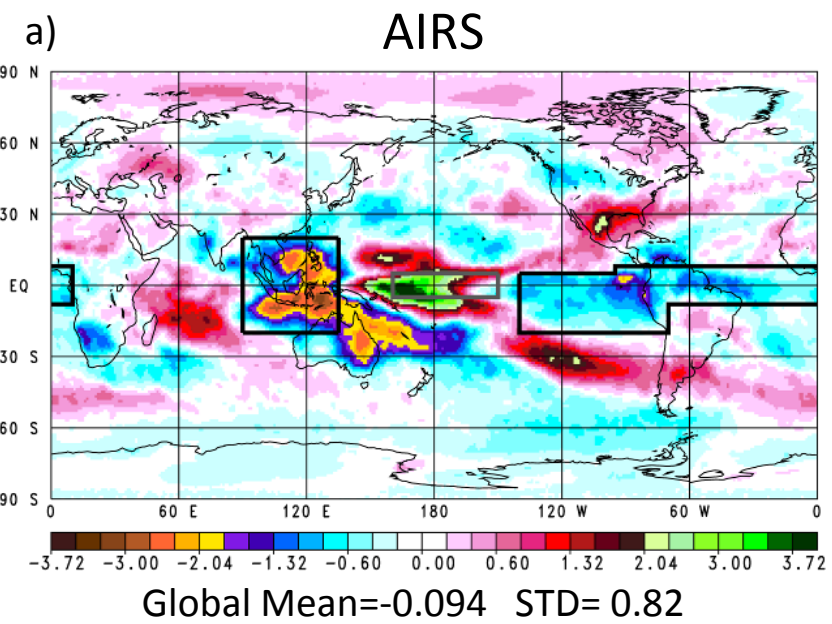

b)

\section{CERES}

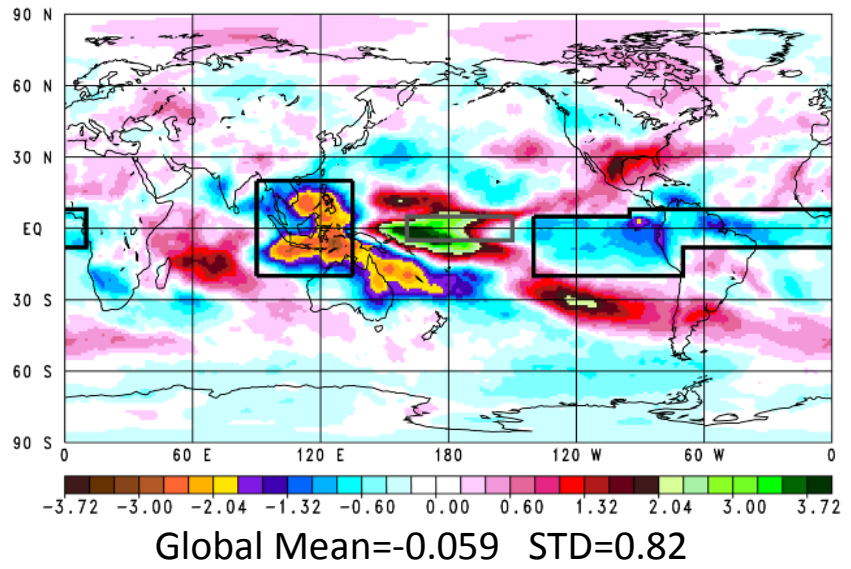

Fig. 3

\section{OLR Anomaly Correlations (ENCs)} September 2002 through June 2011

a)

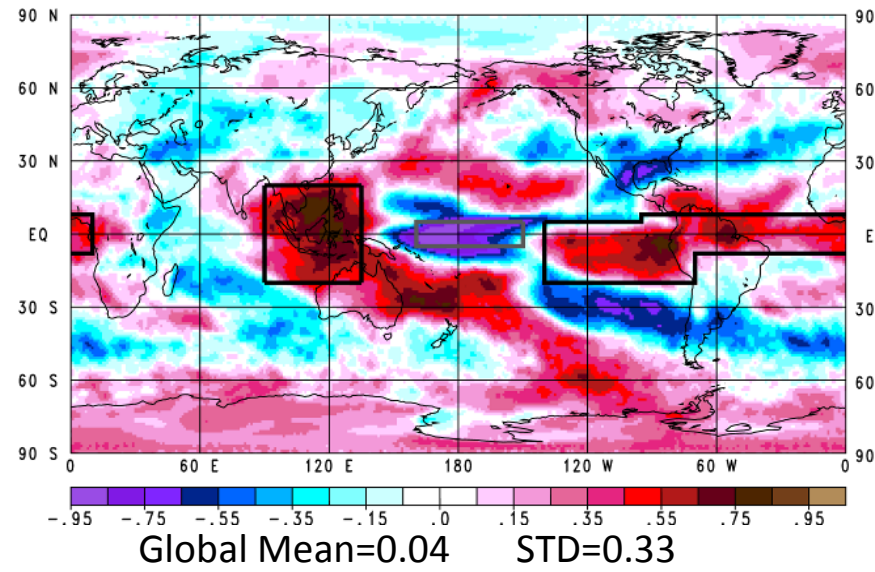

b) CERES

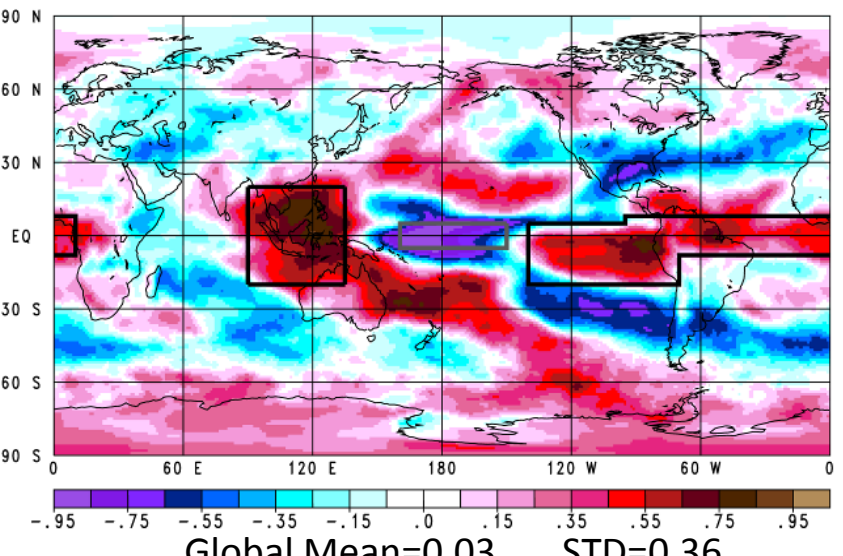

Fig. 4 
region. Indeed, ARCs of OLR within the entire WPMC region are very negative over the period September 2002 through June 2011. ARC's of OLR within the spatially larger EEPA region are also negative over this time period though less so than over the WPMC region. As demonstrated in Figure 3, the ARC of OLR over Darwin, contained in the WPMC region, is opposite in sign to that of Tahiti, near $1 \$$ and $50^{\circ} \mathrm{W}$. As stated previously, tropical OLR will decrease with increasing convective activity, which tends to lead to increased mid-troposphere water vapor and more high clouds on the one hand, and decreased sea level pressure on the other. The reverse phenomena will occur in the case of increasing subsidence in a given region.

Figures 4a,b are analogous to Figures 3a,b but show patterns of ENCs of AIRS and CERES OLR. As found with regard to ARCs of OLR, there is again considerable spatial structure, and excellent agreement, in the ENCs of AIRS and CERES OLR, with a global spatial correlation of 0.97. This agreement shows that not only are the slopes of high spatial resolution anomaly time series of AIRS and CERES OLR in close agreement, but implies that the anomaly time series themselves are also in close agreement. The spatial structure of ENCs of OLR closely follows that of the OLR ARCs but with opposite sign, especially in the tropics. For example, the area of large positive ARCs of OLR including and surrounding the NOAA El Niño region has OLR anomalies which are highly negatively correlated with the El Niño Index, that is, periods of positive SST anomalies in the El Niño region correspond to negative OLR anomalies in this and surrounding areas. The reverse situation is found within the WPMC and EEPA regions, which contain substantial negative OLR ARCs and substantial positive OLR ENCs. This anti-correlation of the spatial distributions of ARCs and ENCs of tropical OLR between September 2002 and June 2011 indicates that the tropical ARCs of OLR shown in Figure 3 are very strongly influenced by time periods containing significant El Niño/La Niña activity. The inverse relationship between spatial patterns of ARCs and ENCs holds in some extra-tropical areas as well. Globally, the spatial distributions of ARCs and ENCs of OLR have correlations of -0.78 and -0.79 for AIRS and CERES respectively.

Table 1 shows that both AIRS and CERES OLR anomaly time series confirm that global mean and especially tropical mean OLR decreased over the time period under study. As depicted in Figure 3, the largest OLR ARCs occur in the tropics within the WPMC region and near the dateline surrounding the El Niño region. OLR ARCs in these areas are roughly equal to each other and of opposite sign. The effects of the large positive and negative tropical OLR ARCs near the dateline and over the WPMC region tend to cancel in the zonal mean sense. The first three lines of Table 2 show values of ARCs and ENCs of OLR averaged over different spatial regions related to the WPMC region, as determined using each of the AIRS and CERES OLR data sets. The first line of Table 2 shows statistics related to ARCs and ENCs of OLR computed only within the WPMC region. AIRS and CERES both show very large negative ARCs and very high

Table 2. Area Mean Statistics for AIRS and CERES OLR September 2002 through June 2011

AIRS

CERES

\begin{tabular}{|l|c|c|c|c|}
\hline \multicolumn{1}{|c|}{ Spatial Area } & $\begin{array}{c}\text { OLR ARC } \\
\left(\mathrm{W} / \mathrm{m}^{2} / \mathrm{yr}\right)\end{array}$ & OLR ENC & $\begin{array}{c}\text { OLR ARC } \\
\left(\mathrm{W} / \mathrm{m}^{2} / \mathrm{yr}\right)\end{array}$ & OLR ENC \\
\hline WPMC Region & $-1.502 \pm 0.525$ & 0.871 & $-1.496 \pm 0.529$ & 0.870 \\
\hline $\begin{array}{l}\text { Tropical outside } \\
\text { WPMC Region }\end{array}$ & $0.004 \pm 0.054$ & 0.050 & $0.034 \pm 0.053$ & 0.101 \\
\hline $\begin{array}{l}\text { Global outside } \\
\text { WPMC Region }\end{array}$ & $-0.030 \pm 0.028$ & -0.129 & $0.005 \pm 0.027$ & -0.372 \\
\hline EEPA Region & $-0.631 \pm 0.158$ & 0.767 & $-0.611 \pm 0.154$ & 0.761 \\
\hline $\begin{array}{l}\text { Tropical outside } \\
\text { EEPA Region }\end{array}$ & $-0.037 \pm 0.048$ & 0.599 & $-0.011 \pm 0.047$ & 0.511 \\
\hline $\begin{array}{l}\text { Global outside } \\
\text { EEPA Region }\end{array}$ & $-0.044 \pm 0.020$ & 0.256 & $-0.011 \pm 0.019$ & 0.039 \\
\hline
\end{tabular}


positive ENCs of OLR within this region. The second and third lines of Table 2 show values of global and tropical mean ARCs and ENCs of OLR computed only over areas outside of the WPMC regions. The negative tropical mean OLR ARC over the period September 2002 through June 2011 computed as previously, but after replacing OLR ARCs in the WPMC region by zeroes, is $0.004 \pm 0.054 \mathrm{~W} / \mathrm{m}^{2} / \mathrm{yr}$ for AIRS, and $0.034 \pm 0.053 \mathrm{~W} / \mathrm{m}^{2} / \mathrm{yr}$ for CERES. Both of these values are essentially zero, given their uncertainties. In addition, ENC's of tropical mean OLR outside of the WPMC region are also both very low. Likewise, the global mean OLR ARC, excluding ARCs within the WPMC region, is $-0.030 \pm 0.028 \mathrm{~W} / \mathrm{m}^{2} / \mathrm{yr}$ for AIRS, and $-0.005 \pm 0.027 \mathrm{~W} / \mathrm{m}^{2} / \mathrm{yr}$ for CERES, again both essentially zero. This indicates that both OLR data sets show that a substantial part of the recent negative global mean and tropical mean OLR ARCs results from the contribution of OLR anomalies contained within the WPMC region to the overall statistics. This statistic can give rise to the misleading conclusion that the response of OLR within the WPMC region is the sole cause of the fact that tropical and global mean OLR anomalies are highly correlated with El Niño/La Niña activity. This is not the case.

Figure 3 shows that OLR ARCs are also very negative over the EEPA region. While ARCs of OLR over the EEPA region are not as negative as those over the WPMC region, the spatial extent of the EEPA region is considerably larger than that of the WPMC region. The last three lines of Table 2 show analogous statistics for OLR within, and outside of, the EEPA region as was shown for the WPMC region. Exclusion of OLR anomalies within the EEPA region likewise accounts for most of the negative ARCs of global and tropical mean OLR found during the period under study. The fact that OLR has been decreasing over the last nine years, and that OLR anomalies are highly correlated with the El Niño Index, is not the result of OLR changes within either of these regions highlighted above. Rather, it is the result of the fact that there are two such regions in the tropics, one to the east and one to the west of the El Niño region, in which OLR anomalies are out of phase with, and individually compensate for, those in the El Niño region.

\subsection{The effect of phases of El Niño/La Niña on tropical water vapor, cloud cover, and OLR anomaly time series}

Equation 2 shows that, everything else being equal, OLR increases with increasing skin temperatures $T_{s}$ and temperature profile $T(p)$, and will generally decrease with increasing water vapor $q(p)$, especially for very moist (i.e. tropical) cases. Equation 1 shows that OLR will in general decrease with increasing fractional cloud cover $\alpha \varepsilon$, especially in the case of high (cold) clouds. It is impractical to show results relating to anomaly time series of all the important geophysical parameters affecting those of OLR. In this paper, for demonstrative purposes, we concentrate on $T_{s}, 500 \mathrm{mb}$ specific humidity $q_{500}$, and total radiatively effective cloud fraction $\alpha \varepsilon$, where $\alpha \varepsilon$ is the sum of $\alpha \varepsilon_{1}$ and $\alpha \varepsilon_{2}$. The OLR calculations of course take into account the detailed changes in the entire water vapor profile and the heights and amounts of clouds, as well as those of the temperature and ozone profiles on which OLR also depends.

Figures $5 \mathrm{a}$ and $5 \mathrm{~b}$ show the spatial distribution of the ARCs and ENCs respectively of AIRS Version-5 $T_{s}$ over the period September 2002 through June 2011. A number of important features are found in Figure 5a. While the global mean ARC of $T_{s}$ is essentially zero over this time period, there are areas in which significant positive and negative $T_{s}$ ARCs exist. There has been considerable warming near the North Pole over this time period, as well as considerable warming and cooling in different areas over Northern Hemisphere extra-tropical land. In addition, there has been substantial cooling over much of Africa, especially south of $15^{\circ} \mathrm{S}$, as well as over much of Australia. All of these areas in which extratropical land has either warmed or cooled considerably over the time period under study are also characterized by increases or decreases in OLR as shown in Figure 3. This is consistent with the fact that everything else being equal, increases (decreases) in $T_{s}$ results in increases (decreases) in OLR. Figure 5a also shows that the tropics are marked by a substantial oceanic surface temperature cooling over and immediately surrounding the El Niño region contained within the gray rectangle. This area of oceanic cooling over the last nine years is surrounded to the south, west, and north by areas in which oceanic warming occurred during this period, though to a lesser extent. Figure 3 shows that OLR changes in these oceanic areas are considerable, and unlike over extra-tropical land, are of opposite sign to those of the changes in $T_{s}$. This indicates that the changes in tropical oceanic OLR in these regions are driven by changes in water vapor profile and cloud cover rather than changes in $T_{s}$.

Figure $5 \mathrm{~b}$ shows that as in the case of OLR, ENCs of $T_{s}$ are generally of opposite sign to their ARCs. The global mean spatial correlation of ARCs and ENCs of $T_{s}$ with each other is -0.56 , which is still appreciable but smaller than that for 
OLR. It is apparent from Figures 5a and 5b that the considerable cooling of $T_{s}$ that took place over South Africa and Australia is related to a strong in phase response of $T_{s}$ to El Niño/La Niña activity in these areas. On the other hand, the significant changes in $T_{s}$ that occurred over Northern Hemisphere extra-tropical land, such as the warming that occurred near the North Pole, are not in direct response to El Niño activity, at least in an unlagged sense, because ENCs of $T_{s}$ in these areas are not appreciable. It is also interesting to note that in some equatorial oceanic areas, such as in the vicinities of $90^{\circ} \mathrm{W}$ and $60^{\circ} \mathrm{E}, T_{s}$ anomalies had moderate positive correlations with El Niño activity but $T_{s}$ in those areas had very small Average Rates of Change. In addition, while ARCs and ENCs of OLR within the WPMC region and the EEPA region were each all large and of the same sign, this is not the case with regard to $T_{s}$.
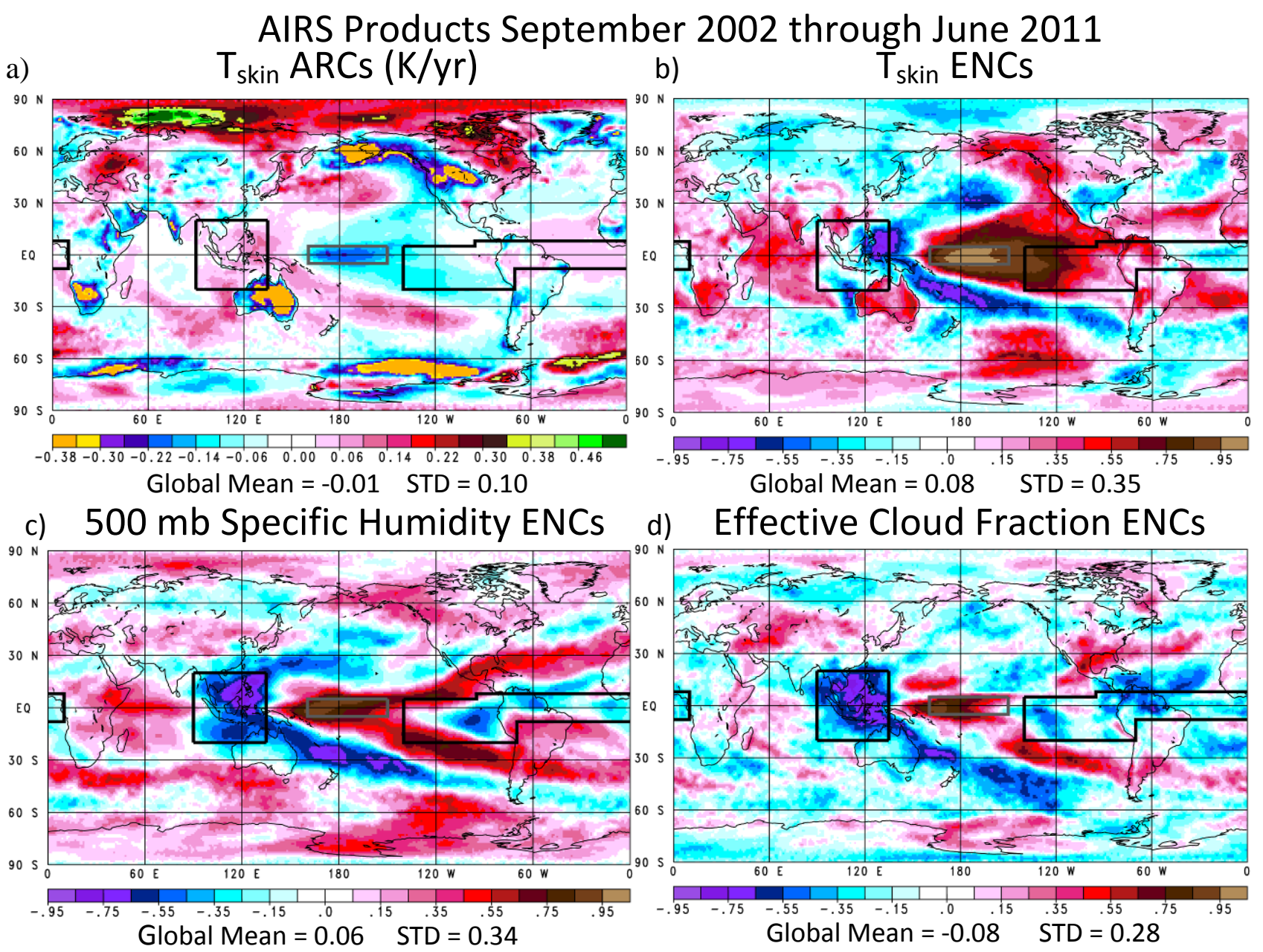

Fig. 5

Figures 5c and 5d show ENCs of $500 \mathrm{mb}$ specific humidity $q_{500}$ and $\alpha \varepsilon$, respectively. In the tropics, ARCs of $q_{500}$ and $\alpha \varepsilon$ (not shown) are out of phase with ENCs of these parameters, as was the case with ARCs and ENCs of OLR. As with $T_{s}$, ENCs of $q_{500}$ and $\alpha \varepsilon$ are both very positive in the El Niño region. This high positive correlation with El Niño activity resulted in both a significant overall mid-tropospheric drying and a corresponding overall decrease in cloud cover in this region over the period under study. An analogous, but opposite, situation occurs in the areas of warming surface skin temperature surrounding the El Niño region, in which ENCs of $T_{s}, q_{500}$, and $\alpha \varepsilon$ all are very negative, resulting in considerable mid-tropospheric moistening as well as increasing cloud cover in these surrounding regions during the period under study. Unlike $T_{s}$ however, there are also large negative ENCs of $q_{500}$ and $\alpha \varepsilon$ contained within most of the WPMC and the EEPA regions over this time period, resulting in both considerable mid-tropospheric moistening and increasing cloud cover over these two regions. Of particular significance are the large negative ENCs of cloud fraction, 
and even more so, $500 \mathrm{mb}$ specific humidity, contained within the EEPA region off the West Coast of South America. These negative ENCs of water vapor and cloud cover, taken together, give rise to the large positive ENCs and large negative ARCs of OLR in this area shown in Figures 4 and 3 respectively.

This study shows that at least over the time period September 2002 through June 2011, during which the El Niño region has cooled, global and tropical OLR have decreased as a result of the combined increases in mid-tropospheric water vapor and cloud cover in each of two regions, one to the east of the El Niño region and one to the west of it. The results shown of this study should not be taken in any way as to be indicative of how OLR will change in the future especially with regard to possible increases or decreases in global mean OLR. The EOS satellites carrying AIRS and CERES are expected to last about 20 years. Having a 20 year time series of overlapping AIRS and CERES OLR data records would be a very useful first step towards monitoring and understanding long term variability of OLR. Continuation of AIRS class and CERES class instrumentation beyond the EOS era is highly desirable to fully monitor and understand longer term changes in OLR.

\section{REFERENCES}

[1] Pagano, T. S., Aumann, H. H., Hagan, D. E. and Overoye, K., "Prelaunch and in-flight radiometric calibration of the Atmospheric Infrared Sounder (AIRS),” IEEE Trans. Geoscience and Remote Sensing, 41, 265-273, DOI: 10.1109/TGRS.2002.808324 (2003).

[2] Susskind, J, Blaisdell, J.M., Iredell, L. and Keita, F., "Improved temperature sounding and quality control methodology using AIRS/AMSU data: The AIRS Science Team Version-5 Retrieval Algorithm,” IEEE Trans. on Geoscience and Remote Sensing, Issue: 99, 1-15, DOI: 10.1109/TGRS.2010.2070508 (2011).

[3] Susskind, J., Barnet, C.D., and Blaisdell, J.M., "Retrieval of atmospheric and surface parameters from AIRS/AMSU/HSB data in the presence of clouds,” IEEE Trans. Geoscience and Remote Sensing, 41, 390-409, DOI: 10.1109/TGRS.2002.808236 (2003).

[4] Chahine, M. T., "Remote sensing of cloudy atmospheres. II. Multiple cloud formations,” J. Atmos. Sci., 34, 744-757 (1977).

[5] Chahine, M. T., "Remote sensing of cloudy atmospheres. I. The single cloud layer,” J. Atmos. Sci., 31, 233-243 (1974).

[6] Reale, O., Lau, W. K., Susskind, J., Brin, E., Liu, E., Riishojgaard, L. P., Fuentes, M., and R. Rosenberg, “AIRS Impact on the Analysis and Forecast Track of Tropical Cyclone Nargis in a Global Data Assimilation and Forecasting System”, Geophys. Res. Let., 36, L06812, doi:10.1029/2008GL037122 (2009).

[7] Zhou, Y. P., Lau, K.-M., Reale, O., and Rosenberg, R., "AIRS Impact on Precipitation Analysis and Forecast of Tropical Cyclones in a Global Data Assimilation and Forecast System," Geophys. Res. Let., 37, L02806, doi:10.1029/2009GL041494 (2010).

[8] Reale, O., Lau, K.M., Susskind, J., and Rosenberg, R., "AIRS impact on analysis and forecast on an extreme rainfall event (Indus River Valley, Pakistan, 2010) with a global data assimilation and forecast system,” J. Geophys. Res., 117, D08103, doi:10.1029/2011JD017093 (2012).

[9] Susskind, J., Blaisdell, J., Iredell, L., "Significant advances in the AIRS Science Team Version-6 retrieval algorithm," SPIE Conference Proc. Paper \#8510-29 (August 2012).

[10] Wielicki, B.A., Barkstrom, B.R., Harrison, E.F., Lee III, R.B., Smith, G.L., and Cooper, J.E., "Clouds and the Earth's radiant energy system (CERES): An Earth observing system experiment,” Bull. Amer. Meteor. Soc., 77, 853868 (1996).

[11] Mehta, A.V. and Susskind, J., “Outgoing longwave radiation from the TOVS Pathfinder Path A data set," J. of Geophys. Res., 104, 12193-12212 (1999).

[12] Susskind J., Molnar, G., Iredell, L., Loeb, N.G., "Interannual variability of OLR as observed by AIRS and CERES,” submitted to J. of Geophys. Res. (2012). 